\title{
RESEARCH PAPER \\ THE POTENTIAL OF PHYSIOLOGICAL ANALYSIS USING ELECTROMYOGRAPHY IN THE DESIGN OF MOTORCYCLES
}

\author{
P. B. Pascolo ${ }^{1}$, C. A. Rossi* ${ }^{1}{ }^{1}$ G. Franceschini ${ }^{2}$, E. M. Zanetti ${ }^{2}$, S. Logozzo $^{2}$ and F. Sciarrini ${ }^{1}$. \\ ${ }^{I}$ Department of Civil Engineering and Architecture - University of Udine, Italy \\ ${ }^{2}$ Department of Engineering, University of Perugia, Italy \\ Corresponding author: E-mail: caterina.rossi@uniud.it
}

\begin{abstract}
Two-wheel vehicle comfort is usually assessed by means of subjective scorings or by measuring physical quantities such as acceleration, sound pressure, etc. which do not depend on the human response. This study has chosen a different approach, which is to use electromyography for evaluating the levels of muscle activity. It focuses more specifically on comfort in relation to aerodynamic loads. The loads were simulated by a wind tunnel. ANOVA statistical analysis was used to establish the impact of aerodynamic loads and of various motorcycle models on muscle activity levels. The results showed that aerodynamic loads generally cause an increase of muscle activity, although their impact varies in relation to the design of the motorcycle: each model of motorcycle produces a specific distribution of activity levels among muscles. The methodology can be used for vehicle-design purposes as well as organising motorbike training programmes.
\end{abstract}

Keywords: Motorcycle, Aerodynamic loads, SEM, Ergonomics, Comfort

\section{INTRODUCTION}

Riding comfort in two-wheel vehicles is a relevant issue since it results in a better driving performance and less fatigue for both the driver and the eventual passenger. The comfort of a vehicle is related to three main aspects (Gameiro da Silva, 2002): dynamic factors such as vibrations and accelerations; environmental factors such as noise, thermal comfort, air quality, visibility etc.; and ergonomic factors, more specifically, the interaction between the driver and the vehicle (through the seat, the handles, etc.). Consequently, various output variables can be referred to as indices of comfort. They can be subjective ratings (scores given by test pilots, filling in specific questionnaires), objective physical variables such as acceleration, vibrations, sound pressures, temperature distribution, or quantitative physiologic measurements. Subjective ratings together with objective physical quantities are the most widely used. The former allow taking into account human subjective reactions and man-machine interactions, while the advantage of measuring 


\section{$51 \quad$ Rossi et al.}

physical quantities is that it provides objective results. Physiological measurements are less common although they can be interesting since they allow for an objective measurement of pilot response. Among the physiological measurements, electromyography can provide information concerning muscle activity in relation to ergonomic aspects (Kumar et al., 1996; AlMulla et al., 2011) through non-invasive index of the levels of muscular activity (Duchene et al., 1993). Many examples can be found in studies concerning sitting biomechanics (Harrison et al., 1999), or comparing various kinds of seats in relation to occupational spine disorders (Makhsous et al., 2009; Nairn et al., 2013).

There are several studies concerning the comfort of 4-wheel vehicle drivers (Albert et al., 2013; Andersson et al., 1974) while studies of rider posture on two-wheel vehicles are less common. Karuppiah et al. (2012) analysed the effect of backrests on the basis of subjective scorings. One limitation of their study was that the motorcyclists were sitting on motorcycles in a controlled environment, and the weight load produced the interface pressures between the men and the vehicles. In order to obtain the most realistic estimation of rider's muscle activity levels, on-the road tests would be required which would be difficult to repeat due to the various conditions such as trajectory, road surface, issues related to driving, meteorological conditions, etc. thus making it difficult to distinguish each specific contribution. A simplified approach, by simulating aerodynamic loads, was used in this study. The study therefore focuses on the rider's posture rather than evaluating other aspects such as manoeuvrability or vibration comfort. A similar approach was followed in the case of other sports where the aerodynamic load is presumed to play an important role with the aim of isolating it from other factors: for example see the studies concerning ski jumping (Virmavirta et al., 2011) or cycling (Chapman et al., 2008) in wind tunnels.

Two different motorcycles were analysed with two different postures: the so-called standard riding posture where the driver has a neutral upright position with his back and neck approximately straight (torso angle is about $17^{\circ}$, knee angle is about $60^{\circ}$, and hip angle is about $91^{\circ}$ according to experimental measurements), and the sport driving posture with forward lean and forward tilt of the pelvis, while the head is in slight extension (torso angle is about $66^{\circ}$, knee angle is about $52^{\circ}$, and hip angle is about $36^{\circ}$ ). The forward leaning, in sport driving, implies the operator is partially supporting his upper body weight on his arms, and the muscles on the back are supporting the head. However, at higher speeds, the weight of the body may be partially supported by air pressure rushing onto the torso for bike riders (Blocken et $a l ., 2013$ ) or it might get even worse, depending on the boundary layer as determined by the windshield. The standard riding posture is perceived as more comfortable by riders (see the optimal angles reported by Chou and Hsiao, 2005).The larger rider frontal area increases drag resistance (Blocken et al., 2013), therefore a higher muscle work is required in order to counterbalance aerodynamics loads.

The information on muscular stress caused by various motorcycling postures can be useful for designing new vehicles as well as for drawing up specific training protocols for athletic competitors.

\section{MATERIALS AND METHODS Participants}

Six riders were chosen and they gave their written informed consent to the experiments. They were all male, aged $42 \pm 5$ years, whose mass was equal to $78 \pm 8 \mathrm{~kg}$, their height was equal to $178.0 \pm 8.5 \mathrm{~cm}$, and their BMI was equal to $24.25 \pm 2.9 \mathrm{~kg} / \mathrm{m} 2$. They were all equipped with the same sensors. Each subject had anthropometric characteristics that closely matched the 50th percentile MATD dummy used for ISO 13232 testing. All the riders participating in the experiment had the same lifestyle: they were healthy, they did not use drugs and they were not addicted to alcohol. They were frequent 
motorcycle users, and therefore they were able to establish if their vehicle was comfortable at first instance. A preliminary questionnaire was submitted to all subjects.

\section{Materials \\ Aerodynamic loads were simulated by means of the wind tunnel located at the Department of Engineering of the University of Perugia (2200x2200 $\mathrm{mm}^{2}$; Fig.1). Two different vehi- cles were considered (Fig. 2). The first vehicle is a sport touring naked motorcycle. This vehi- cle provides minimal wind protection for the rider and its experimentally measured drag co- efficient is equal to 0.61 . It is designed for short trips out of town. The driving posture is moder- ately bent forward. The second vehicle is a high performance sport bike. The driving posture is aimed at obtaining the best possible perform- ance of the vehicle in spite of comfort of the driver. The driver must be leaning forward in order to be protected from the wind. The ex- perimentally measured drag coefficient is equal to 0.28 . This vehicle was specifically designed for competitions on racetracks.}

These models were chosen because they represent motorcycles designed for different purposes and requiring different driving postures. They were put at our disposal by the kind concession of their manufacturer. The speed of the air was equal to $43 \mathrm{~m} / \mathrm{s}$. This high speed was chosen in order to maximize the aerodynamic load contribution, and it was considered a possible top speed on the slower sport touring motorcycle model.

\section{Methods}

Surface electromyography was carried out with a 16 channels device (PocketEMG ${ }^{\mathrm{TM}}$ by BTS). The sensors were positioned carefully in order to achieve a high level of repeatability, with a distance of $20 \mathrm{~mm}$ between the electrodes. The following muscles were monitored bilaterally: the biceps, the triceps, and the upper trapezius, the pectoralis major; the electrodes were applied over the muscle bellies, after shaving, scrubbing and cleaning the skin with alcohol.
For analysing the upper trapezius, the electrodes were placed at a point two-thirds of the distance from the spine of the seventh cervical vertebra $(\mathrm{C} 7)$ to the lateral edge of the acromion (Jensen et al. 1993). Electrodes for the biceps and triceps were located on the muscle bellies, and the electrodes of the pectoralis major (sternal fiber) were placed on the chest wall, horizontal from the rising muscle mass (approximately $2 \mathrm{~cm}$ out from the axillary fold).

The EMG activity has been digital band-pass filtered at $10-500 \mathrm{~Hz}$; it was sampled at $1 \mathrm{kHz}$, and digitised at 16 bits, in compliance with recommendations from European SENIAM (Surface Electromyography for the NonInvasive Assessment of Muscles) project (Stegeman and Hemens, 2012). Data analysis was performed by means of Myolab software.

The riders underwent various tests before data acquisition in order to get them acquainted with the instrumentation; each test lasted $75 \mathrm{sec}$ onds. During each stationary phase (with/ without wind), the average RMS of each EMG signal (ARMS) was calculated.

The statistical analysis of data was performed by means of two-way within subjects ANOVA, after having checked the normality of ARMS data distribution, and their Sphericity' (Norman et al., 2008), and established a level of confidence equal to $95 \%(\mathrm{p}<0.05)$. Two factors were considered: the first factor was the model of the motorcycle on two levels: the sport touring motorcycle or the sport bike. The second factor was the aerodynamic load; this factor also has two levels: 0 wind speed and $43 \mathrm{~m} / \mathrm{s}$ wind speed. All riders performed all four tests (2 motorbikes x 2 aerodynamic load levels), in a randomized order. 

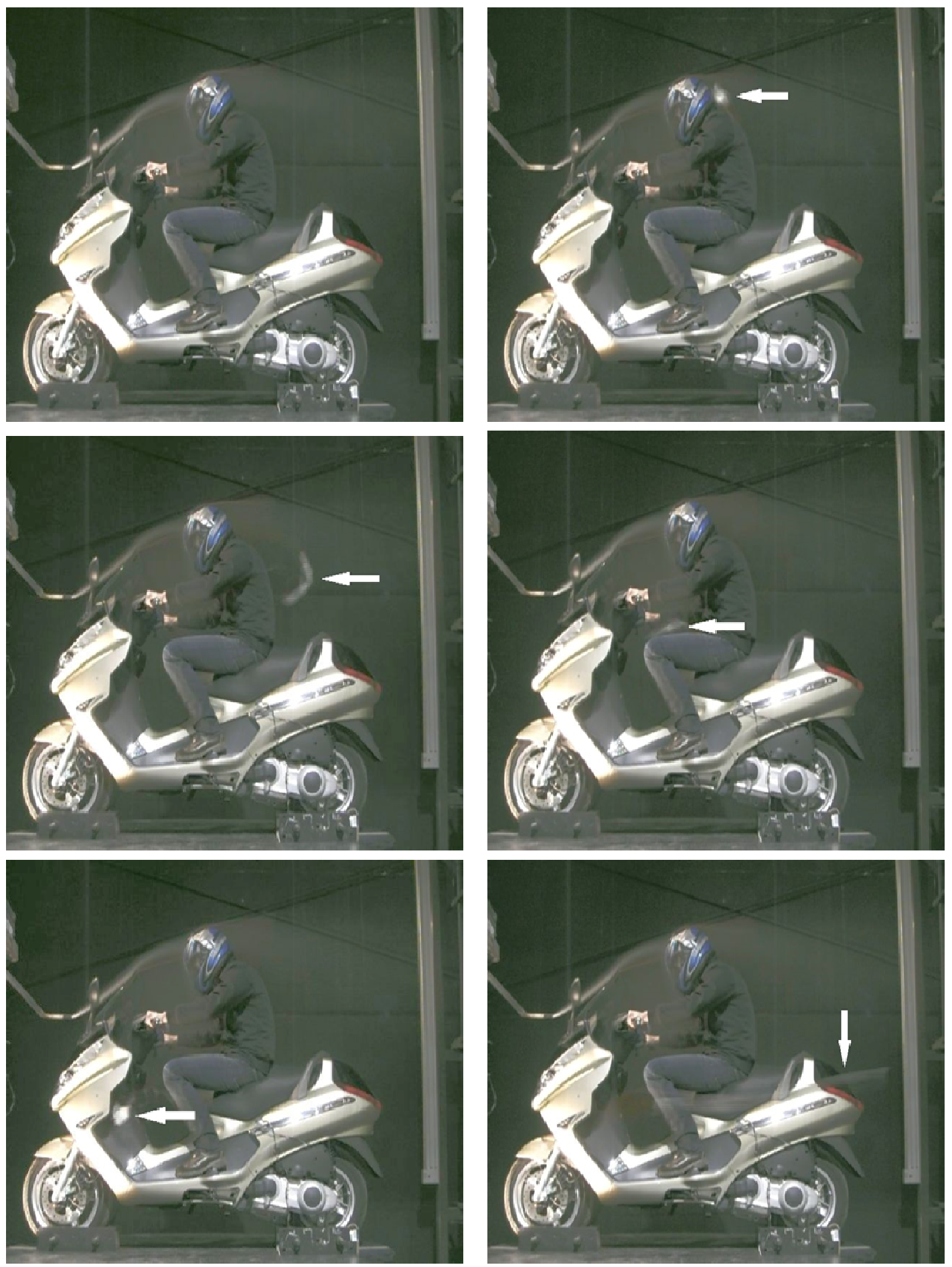

Fig. 1: Sequence in wind-tunnel (arrows show the streamline at $90 \mathrm{~km} / \mathrm{h}$ )

Journal of Science and Technology @ KNUST August 2016 

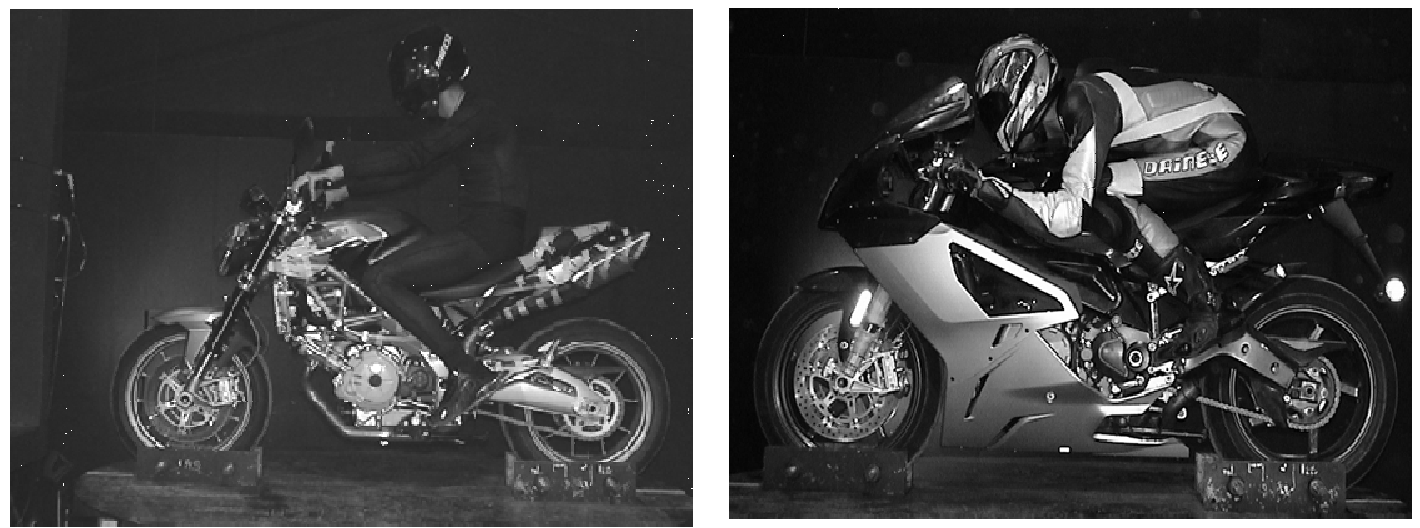

Fig. 2 : Motorcycles used in the tests: sport touring naked motorcycle (left); high performance sport bike (right); note the differences between driver postures (photos published courtesy of the Piaggio group)
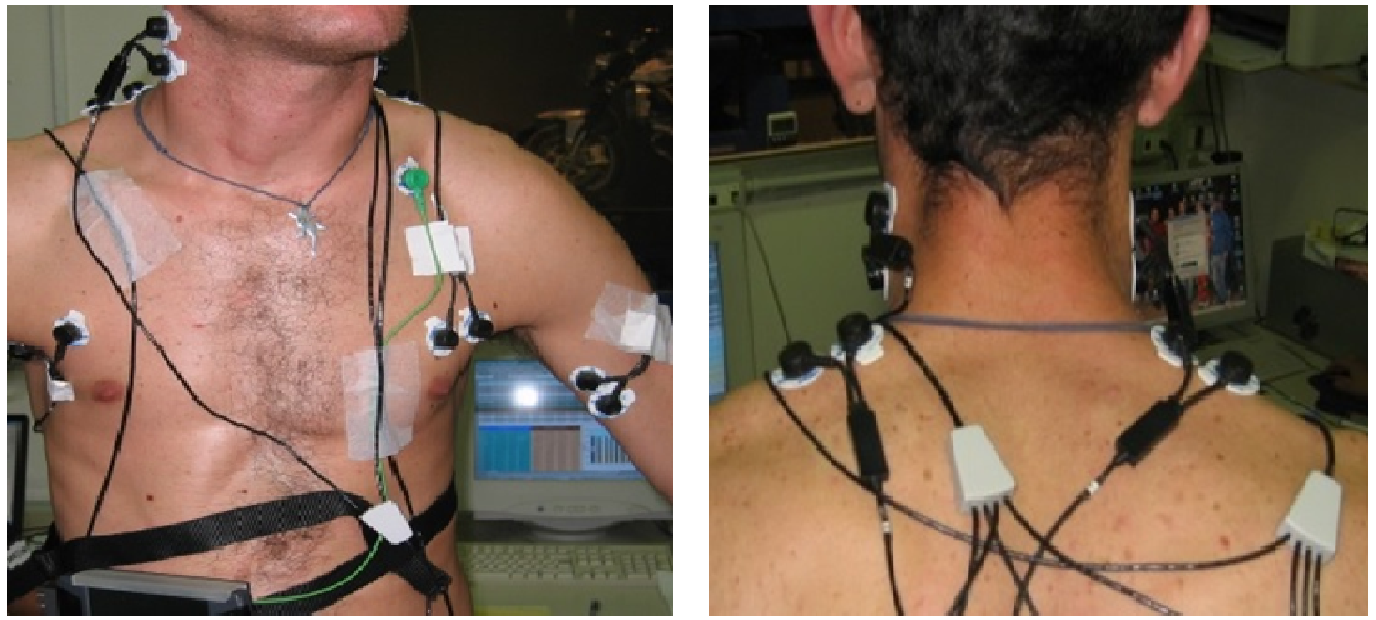

Fig. 3: Example of EMG electrodes positioning on a test rider

\section{RESULTS AND DISCUSSION}

ANOVA Results

Fig. 4, Table 1 and Table 2 shows the results of experimental tests.

ANOVA analysis has been reported in Table 2. For all muscles, the influence of motorcycle model and of the wind is significant as well as their interaction.

\section{Evaluation of muscle activity}

The peculiar pattern of muscle activity during blowing in the wind tunnel has been reported in Fig. 5. 
Fig. 4: EMG results; differential readings are given with reference to the respective muscle activity of each rider on the sport touring motorcycle, in the absence of wind

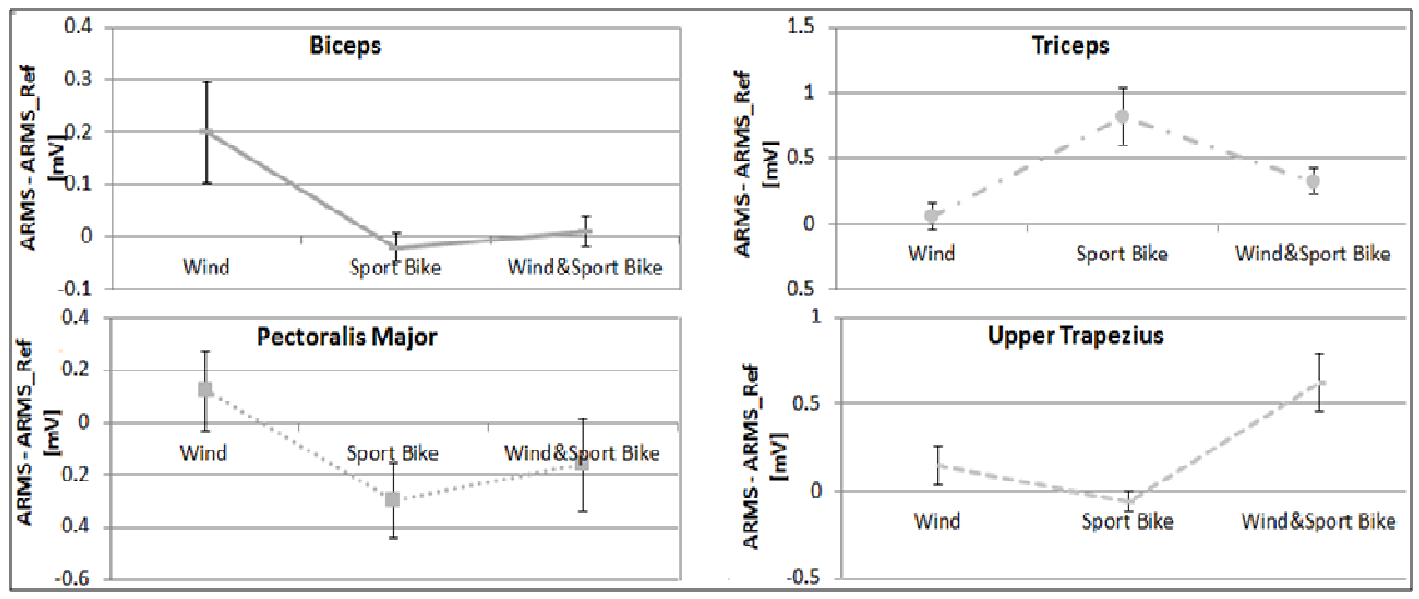

\section{Upper arm muscles}

Both the presence of wind and the motorcycle design proved to influence muscle activation levels. There was also a significant interaction between these two factors. More in detail, both the biceps and the triceps activation levels more often increased in the presence of wind: aerodynamic loads make trunk control more difficult, and both upper arm muscles are activated in order to guarantee stability. In the presence of wind, the biceps activation level was higher on the sport touring motorbike, probably due to a tendency of the trunk to rotate backwards as an effect of the pressure difference between the trunk and the back. In the absence of wind, differences in the biceps activation levels were not significant. Opposite trends were observed regarding triceps activation levels in the presence of wind: the muscle activation level was higher on the sport bike than the touring bike due to the tendency of the trunk to lean forward. It is interesting to note that the triceps activation level was higher on the sport bike in the absence of wind and therefore aerodynamic loads play a beneficial role as they help to support the rider's weight. The triceps showed the highest level of activation, which occurred on the sport bike without aerodynamic loads. This is a reason of concern since it can lead to a higher level of fatigue for the rider when travelling at moderate speed.

\section{Upper trapezius and major pectoralis}

Both the presence of wind and the model of the motorcycle proved to influence the upper trapezius and pectoralis major activation levels. The interaction between the wind and the motorcycle model only appeared to be relevant for the upper trapezius.

Both muscle activity levels increase in the presence of wind: aerodynamic loads make neck control more difficult and both muscles are activated in order to guarantee its stability. In the presence of wind, the upper trapezius activation level is higher on the sport bike, probably due to the tendency of the neck to bend downwards. In the absence of wind, the activation level of this muscle is low for both motorcycle models, and no significant differences were observed. Opposite trends can be observed concerning the pectoralis major activa- 


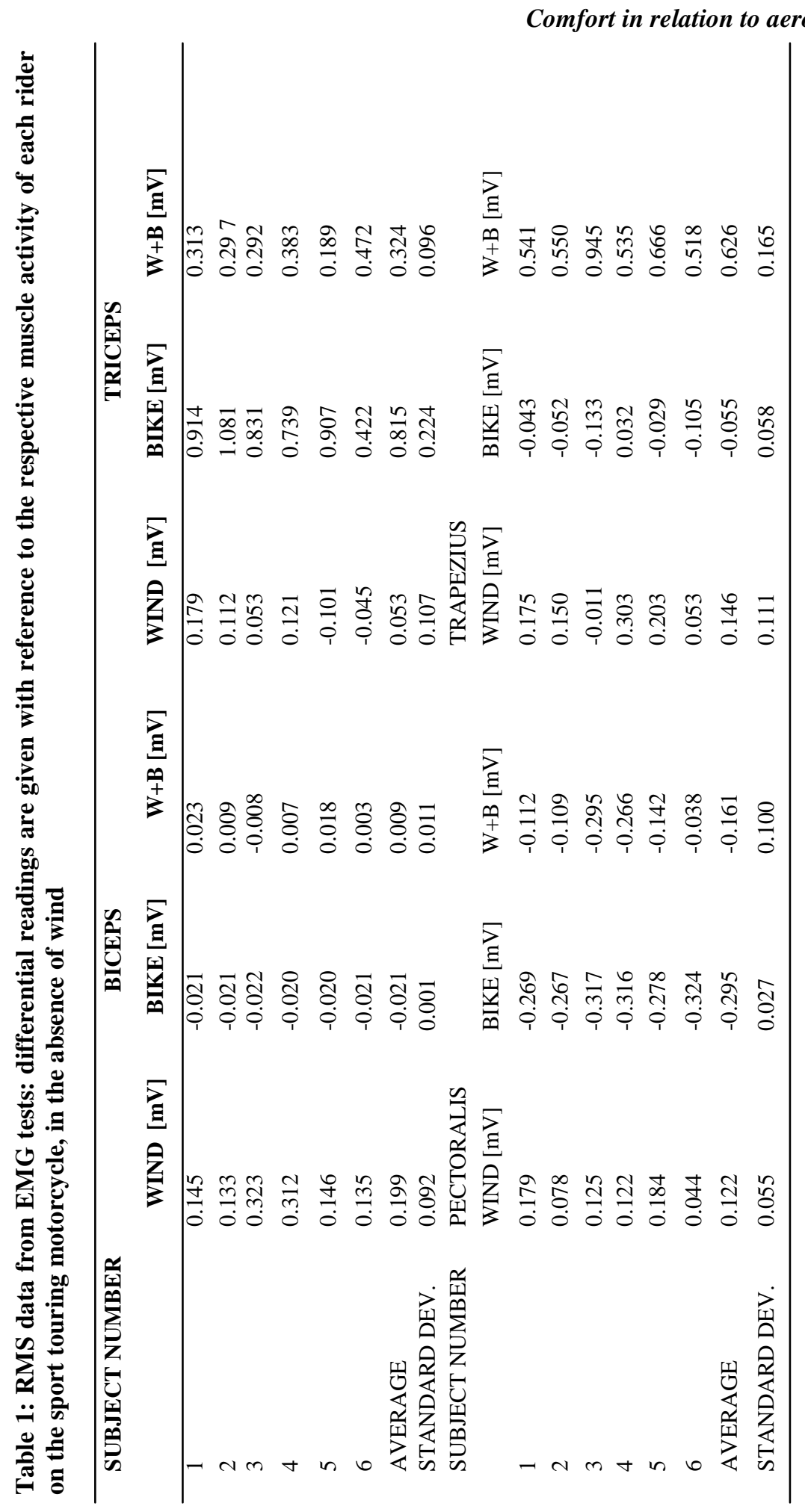

Journal of Science and Technology @ KNUST August 2016 
Comfort in relation to aerodynamic loads... 58

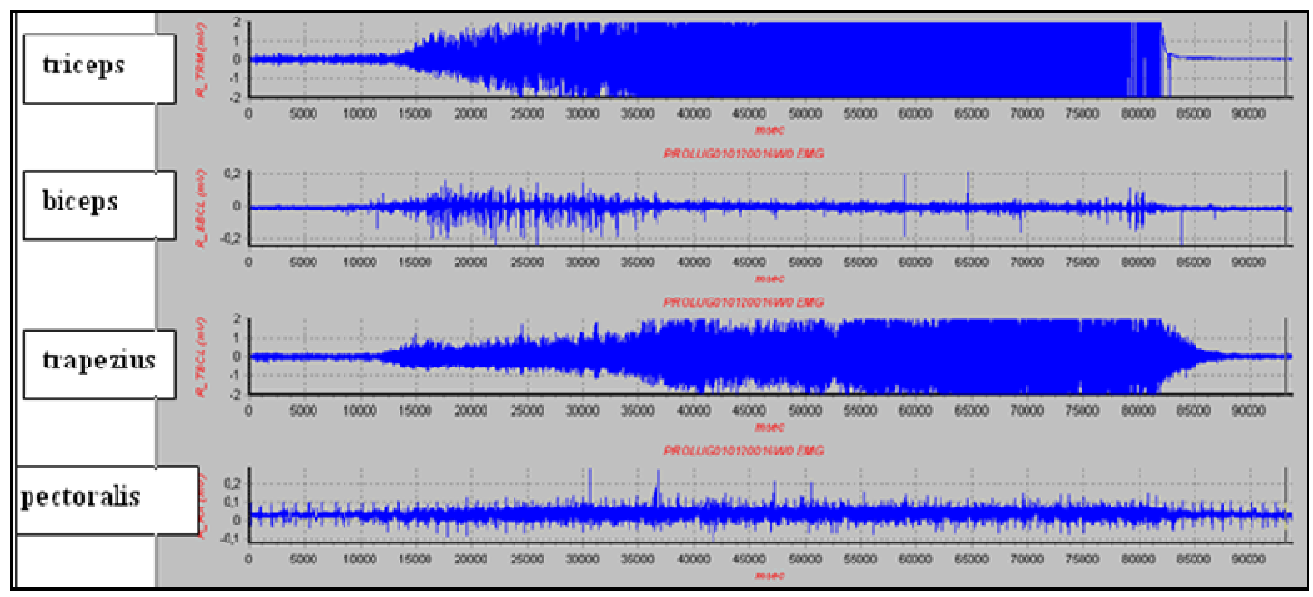

Fig. 5: Sample patterns of muscle activity during the blowing in the wind-tunnel $(0-140 \mathrm{~km} / \mathrm{h})$

tion level in the presence of wind. This muscle activation level is higher on the sport touring motorcycle, probably due to a greater tendency of the neck to rotate backwards. In the absence of wind, the highest activation level of the pectoralis can still be observed on the sport touring motorcycle; therefore, the interaction between the wind and the motorcycle model did not prove to be significant.

\section{CONCLUSIONS}

EMG data could be normalised by referring to the maximum voluntary contraction (MVC), as carried out in most studies in literature. However, the reference contraction to be used for EMG amplitude normalization would be controversial as can be seen in the observations concerning the definition of reference contractions in cycling (Fernández-Peña et al., 2008). The level of activation of neck and upper limb muscles is relevant since the bio-mechanical workload proved to play an important role in musculoskeletal disorders which cause pain and functional impairment in the neck and shoulders (see, for example, studies concerning work -related musculoskeletal disorders described by Buckle et al., 2002). Moreover muscle fatigue can have an effect on safety (Strahan et al.,
2008). A reduction in exposure to muscle-pain risk factors and fatigue can be achieved by following an ergonomic redesign (Keyserling et al., 1993).The protocol introduced in this study could be useful as it allows for the measurement of objective physiological quantities that are directly related to human response.

This study presents a scientific protocol for analysing ergonomics in relation to aerodynamic loads with reference to two-wheeled vehicles. This protocol yields objective physical measurements describing human response and, more specifically, muscle activation levels. The protocol proved to be effective in determining the performance of various motorcycle models concerning comfort and assessing the impact of aerodynamic loads. The results concerning muscle activation levels can be useful information for ergonomic design and for drawing up specific training programmes, in order to alleviate muscle fatigue thus improving safety issues.

\section{FUTURE DEVELOPMENTS}

This study wants to focus on future research on neck muscles stress of the rider in order to create bikes that are more comfortable for riders 
themselves.

\section{ACKNOWLEDGEMENT}

We wish to thank the Aprilia and Piaggio Group for providing the vehicles used in the tests and for their permission to publish the photographs and disclose the research results.

\section{REFERENCES}

Albert, W. J., Everson, D., Rae, M., Callaghan, J. P., Croll, J. and Kuruganti, U. (2013). Biomechanical and ergonomic assessment of urban transit operators. Work. doi: 10.3233/ WOR-131683 2013.

Al-Mulla, M. R., Sepulveda, F. and Colley, M. (2011). A Review of Non-Invasive Techniques to Detect and Predict Localised Muscle Fatigue. Sensors, 11, 3545-3594; doi:10.3390/ s110403545

Andersson, B. J., Ortengren, R., Nachemson, A. and Elfström, G. (1974). Lumbar disc pressure and myoelectric back muscle activity during sitting. IV. Studies on a car driver's seat. Scandinavian Journal of Rehabilitation Medicine, 6: 128-133.

Blocken, B. J. E., Defraeye, T.W.J., Koninckx, E., Carmeliet, J. E. and Hespel, P. (2013). CFD simulations of the aerodynamic drag of two drafting cyclists. Computers and Fluids, 71: 435-445.

Buckle, W. J. and Devereux, J. J. (2002). The nature of work-related neck and upper limb musculoskeletal disorders. Applied Ergonomics, 33: 207-217.

Chapman, A. R., Vicenzino, B., Blanch, P., Knox, J. J., Dowlan, S. and Hodges, P. W. (2008). The influence of body position on leg kinematics and muscle recruitment during cycling. Journal of Science and Medicine in Sport, 11: 519-526.

Chou, J. R. and Hsiao, S. W. (2005). An anthropometric measurement for developing an electric scooter. International Journal of Industrial Ergonomics, 35: 1047-1063.

Duchêne, J. and Goubel, F. (1993). Surface electromyogram during voluntary contraction: processing tools and relation to physiological events. Critical Revues in Biomedical Engineering, 21: 313-397.

Fernández-Peña, E., Lucertini, F. and Ditroilo, M. (2008). A maximal isokinetic pedalling exercise for EMG normalization in cycling. Journal of Electromyography and Kinesiology, 19: 162-170.

Gameiro da Silva, M. C. (2002). Measurements of comfort in vehicles. Measurement Science and Technology, 13, R41-R60.

Harrison, D. D., Harrison, S. O., Croft, A. C., Harrison, D.E. and Troyanovich, S. J. (1999). Sitting Biomechanics Part I. Journal of Manipulative and Physiological Therapeutics, 22: 594-609.

Jensen, C., Vasseljen, O. and Westgaard, R. H. (1993). The influence of electrode position on bipolar surface electromyogram recordings of the upper trapezius muscle. European Journal of Applied Physiology, 67: 266 -273 .

Karuppiah, K., Salit, M. S., Ismail, M. Y., Ismail, N. and Tamrin, S. B. (2012). Evaluation of motorcyclist's discomfort during prolonged riding process with and without lumbar support. Anais da Academia Brasileira de Ciencias, 84: 1169-88.

Keyserling, W. M., Brouwer, M. L. and Silverstein, B.A. (1993). The effectiveness of a joint labour-management program in controlling awkward postures of the trunk, neck, and shoulders: results of a field study. International Journal of Industrial Ergonomics, 11: $51-65$

Kumar, S. and Mital, A. (1996). Electromyog- 
Comfort in relation to aerodynamic loads... 60

raphy in Ergonomics. London: Taylor and Francis.

Makhsous, M., Lin, F., Bankard, J., Hendrix, R. W., Hepler, M. and Press, J. (2009). Biomechanical effects of sitting with adjustable ischial and lumbar support on occupational low back pain: evaluation of sitting load and back muscle activity. BMC Musculoskeletal Disorders, 10, 17. doi: 10.1186/1471-247410-17

Nairn, B. C., Azar, N. R. and Drake, J. D. (2013). Transient pain developers show increased abdominal muscle activity during prolonged sitting. Journal of Electromyography and Kinesiology, 23: 1421-1427.

Norman, G. R. Streiner, D. L. (2008). Biostatistics the Bare Essentials. Shelton, Connecticut, USA: People's Medical Publishing House.
Stegeman, D. F. and Hermens, H. J. (2012). Standards for surface electromyography: the European project "surface EMG for noninvasive assessment of muscles (seniam)." h t t p s :// w w w.res earchgate.net/ $\begin{array}{lllllllll}\mathrm{p} & \mathrm{u} & \mathrm{b} & \mathrm{l} & \mathrm{i} & \mathrm{c} & \mathrm{a} & -\end{array}$ tion /228486725_Standards_for_surface_elec tromyography_the_european_project_surface _emg_for_noninvasive_assessment_of_musc les_\%28seniam\%29.

Strahan, C., Watson, B. and Lennon, A. (2008). Can organisational safety climate and occupational stress predict work-related driver fatigue? Transportation Research Part F, 11, 418-426.

Virmavirta, M., Kivekäs, J. and Komi, P. (2011). Ski jumping takeoff in a wind tunnel with skis. Journal of Applied Biomechanics, 27: $375-379$. 